\title{
Myo Bileklik İle Gerçek Zamanlı Protez Kol Kontrolü
}

\author{
Cengiz Tepe ${ }^{1}$, Muhammed Erdim ${ }^{2 *}$, İlyas Eminoğlu ${ }^{3}$ \\ ${ }^{1}$ Ondokuz Mayıs Üniversitesi, Mühendislik Fakültesi, Elektrik Elektronik Mühendisliği Bölümü, Samsun, Türkiye (ORCID: 0000-0003-4065-5207) \\ 2 Ondokuz Mayıs Üniversitesi, Lisansüstü Eğitim Enstitüsü, Elektrik Elektronik Mühendisliği Anabilim Dalı, Samsun, Türkiye (ORCID: 0000-0001-7467-3249) \\ ${ }^{3}$ Ondokuz Mayıs Üniversitesi, Mühendislik Fakültesi, Elektrik-Elektronik Mühendisliği Bölümü, Samsun, Türkiye (ORCID: 0000-0003-4143-1395)
}

(Bu yayın 26-27 Haziran 2020 tarihinde HORA-2020 kongresinde sözlü olarak sunulmuştur.)

(DOI: $10.31590 /$ ejosat.779672)

ATIF/REFERENCE: Tepe, C., Erdim, M. \& Eminoğlu, İ. (2020). Myo Bileklik İle Gerçek Zamanlı Protez Kol Kontrolü. Avrupa Bilim ve Teknoloji Dergisi, (Special Issue), 184-193.

\section{$\ddot{O} \mathbf{z}$}

İnsan vücudundaki hareketleri algılamayı sağlayan giyilebilir cihazların kullanımı teknolojinin gelişmesi ile birlikte artmaktadır. Çalışma kapsamında Thalmic Labs tarafından üretilen myo kol bandı ile protez kol kontrolü gerçek zamanlı olarak gerçekleştirilmiştir. İnsan kolundaki kasların kasılması sonucunda myo bileklikteki sensorlar ile EMG (elektromiyogram) ve IMU(Atalet Ölçüm Ünitesi) verileri alınmıştır. Bu veriler ile dört el hareket sınıf bilgisi oluşturulmuştur. Bu sınıf bilgileri HM 10 Bluetooth Modülü ile myo bileklikten arduinoya doğrudan aktarılmıştır. Bu çalışmada protez kol için el, bilek, dirsek ve omuz kontrolü için ilk kez anahtarlama mod yöntemi kullanılmıștır. Ampute bireylerde etkin kas sayısı yetersiz olduğundan dolayı az sayıda olan kaslardan alınan EMG verileri ile çok az sayıda protez kol hareketleri denetiminin gerçekleştirilmesi önemlidir. Bu çalışmada oluşturulan sistem ampute bireylerdeki bu hareket kısıtlamalarına bir çözüm niteliğindedir.

Anahtar Kelimeler: Elektromiyogram (EMG), Protez Kol, Myo Kol Bandı, Anahtarlama Mod Yöntemi.

\section{Real Time Prosthesis Arm Control With Myo Armband}

\begin{abstract}
The use of wearable devices, which provide perception of movements in the human body, is increasing with the development of technology. Within the scope of the study, prosthetic arm control was performed in real time with the myo armband produced by Thalmic Labs. As a result of contraction of the muscles in the human arm, EMG (electromyogram) and IMU (Inertial Measurement Unit) data were obtained with sensors on the myo wrist. With these data, four hand motion class information was created. This class information was directly transferred from the myo wristband to the arduino with the HM 10 Bluetooth Module. In this study, for the first time, switching mode method was used for hand, wrist, elbow and shoulder control for the prosthetic arm. It is important to perform a very small number of prosthetic arm movements with EMG data obtained from a small number of muscles due to the insufficient number of effective muscles. The system created in this study is a solution to these movement restrictions in amputated people.
\end{abstract}

Keywords: Electromyogram (EMG), Prosthetic Arm, Myo Armband, Switching Mode Method.

\section{Giriş}

Hayatın her alanında kullanılabilen giyilebilir teknolojik cihazlar, insanlara çeşitli kolaylıklar sağlamakta ve yaşam kalitesini artırmaktadır. Teknolojik gelişmelere bağlı olarak değişime uğrayan bu cihazların oyun, otomotiv, endüstri, güvenlik ve sağlık sektörlerinde kullanımı yaygınlaşmaktadır(Uzunhisarcıklı, Çetinkaya, Fidan, \& Çalıkuşu, 2019). Giyilebilir teknolojik cihazlardan bir tanesi de myo kol bandidır.

\footnotetext{
* Sorumlu Yazar: Ondokuz Mayıs Üniversitesi, Lisansüstü Eğitim Enstitüsü, Elektrik Elektronik Mühendisliği Anabilim Dalı, Samsun, Türkiye, ORCID: 0000-0001-7467-3249, merdim5552@gmail.com
} 
Literatür incelendiğinde Myo kol bandı kullanılarak yapılan çeşitli araştırmalar olduğu görülmektedir. Bu çalışmalar Elektromiyogram(EMG) sinyalinin sinıflandırlması (Boyali \& Hashimoto, 2016; Cognolato, Atzori, Faccio, et al., 2018), robot kol kontrolleri(Erin \& Boru, 2018; Gelen \& Özcan, 2019), multimedya kontrolleri(Klein, 2015), biyonik kol kontrolü(Said et al., 2019), sanal platform kontrolleri(Ganiev, Shin, \& Lee, 2016; Tabor et al., 2016), insansız araç kontrolleri (Cacace, Finzi, \& Lippiello, 2016; Wopereis, Fumagalli, Stramigioli, \& Carloni, 2015) olarak sınıflandırılabilir. Ayrıca myo kol bandı tıbbı çalışmalarda da kullanılmıştır(Sathiyanarayanan \& Rajan, 2016).

Erin ve ark. (Erin \& Boru, 2018) myo kol bandı ile endüstriyel robot kolu kontrol etmeyi amaçlayan çalı̧malarında robot kolu kontrol etmek için ara yüz oluşturmuşlardır. Myo kol bandından bilgisayara, bilgisayardan da robot kola veri aktarımı yapmışlardır. EMG ve jiroskop verilerini kullanarak IRB120 endüstriyel robotu gerçek zamanlı kontrol etmişlerdir. Başka bir çalışmada Gelen ve ark. (Gelen \& Özcan, 2019) myo bileklik kullanarak insanın kol hareketleri ile robot kolun gerçek zamanlı kontrolü yapılmıştır. Omuz, pazı ve ön kola algılayıcı yerleştirerek matematik modelleme oluşturmuş konum kontrolü gerçekleştirilen çalışmada tutucu kontrolü için myo kol bandını kullanılmıştır. Ganiev ve ark. (Ganiev et al., 2016) myo kol bandı içerisinde bulunan EMG, jiroskop ve ivmeölçer sensorlarından aldıkları verileri bluetooth aracılığıyla bir bilgisayara göndererek bu verileri Unity 3D de sanal bir platformda robot kolu kontrol etmişlerdir. EMG ve IMU(Atalet Ölçüm Ünitesi) sensorları kullanarak kol hareketlerini el hareketlerinden ayırmayı amaçladığı çalışmada Lopes ve ark. (Lopes et al., 2017) IMU ile kol hareketlerinin EMG ile el ve parmak hareketlerinin verilerini almışlardır. Çoban ve ark. (Çoban \& Gelen, 2018) myo kol bandı kullanarak robot kol kontrol etmişlerdir. Robot kol ünitesi ethernet üzerinden iletişim kurmaktadır. Raspberry Pi 3 ile robot arasındaki iletişimi TCP/IP protokolü kullanılarak yapmışlardır. Raspberry Pi 3 kullanılarak kod, python dilinde yazılmıştır. Diğer bir çalışmada ise Ülkir ve ark. (Ülkir, Akgün, \& Kaplanoğlu, 2017) myo kol bandı ile kas kasılmaların kullanarak 4 serbestlik derecesine sahip robotu kontrol etmişlerdir. Rasperry pi kullanarak bluetooth üzerinden myo bileklik ile haberleşmişlerdir. Robot üzerinde açma kapama yapacak şekilde bulunan kıskacın kontrolünü gerçekleştirmişlerdir. Said ve ark. (Said et al., 2019) myo bileklikte tanımlanan kalibrasyon ara yüzü ile biyonik kol kontrolü gerçekleştirmişlerdir. Chestnut board kartını kullanmışlardır. Bu kartın üzerinde 4 adet motor bulunmaktadır. Myo verilerini ara yüz ile karta aktarmışlardır ve biyonik kolun kontrolünü gerçekleştirmişlerdir. Kristof ve ark. (Kristof, Moldovan, Ciupe, Maniu, \& Banda, 2019) myo kol bandında bulunan EMG sensorlarını kullanarak iki farklı çalışma yapmışlardır. İlk çalışma, kullanıcının sadece bir kolunu hareket ettirerek bir robotun tutucu kontrolü için insan makine arabirimi (HMI) kullanılmışlardır. İkinci uygulama ise, arduino ile mobil robotun gerçek zamanlı kontrolüdür. Myo kol bandı ile bluetooth kullanarak bilgisayara veri aktarmışlardır. C++ programı kullanmışlardır. RS232 seri bağlantısını kurarak KUKA KR 15 endüstriyel robotunu kontrol etmişlerdir. Morais ve ark. (Morais, Neves, Masiero, \& de Castro, 2016) myo kol bandını kullanarak PeopleBot olarak adlandırılan robotu myoelektrik ve IMU(Atalet Ölçü Birimi) ile kontrolünü gerçekleştirmiş̧lerdir. Cognolato ve ark. (Cognolato, Atzori, Faccio, et al., 2018) el ampütasyonu bulunan üç kişiden myo kol bandı kullanarak beş farklı el hareketinin EMG verilerini almışlardır. Bu verileri kullanarak sınıflandırma yapmışlardır. Her birey için ayrı ayrı sınıflandırmışlar ve \%50 ile \%97 arasında değişen sınıflandırma sonuçlarına ulaşmışlardır. Xu ve ark. (Xu, Yang, Liu, \& Li, 2018) mobil robot kontrol etmişlerdir. Robotun hareket yönünü myo bileklikten alınan EMG sinyallerini sınıflandırarak gerçekleştirmiş̧lerdir. Dört el hareketini sınıflandırmışlardır. Sınıflandırmada SVM (Support Vector Machine) yöntemini kullanarak mobil robot kontrolünü gerçekleştirmiş̧lerdir. Murillo ve ark. (Murillo \& Moreno, 2016) myo bileklikte tanımlı beş el hareketini kullanarak iki manipülatör kolu kontrol etmiş̧lerdir. İlk manipülatörde arduino uno ve dört adet servo motor kullanılmıştır. Diğer manipülatörde ise arduino mega ve yedi adet servo motor kullanılmıştır. Hassan ve ark. (Hassan, Abou-Loukh, \& Ibraheem, 2019) myo bileklik kullanarak yedi el hareketini sınıflandırmış ve beş eksenli Aideepen ROT3U robot kolunu gerçek zamanlı olarak kontrol etmişlerdir. Veri seti üzerinde en yüksek doğruluk KNN ile sağlanmıştır. Robot kolu kontrol etmişlerdir. Yüzgeç ve ark. (Yüzgeç, Büyüktepe, \& Karakuzu, 2016) kablosuz eldiven sistemi ile robot kontrolü gerçekleştirmişlerdir. Özel bir eldiven tasarlamış ve eldiven üzerine eğim sensorları yerleştirmişlerdir. Kablosuz eldiven ile robot kol kontrolü yapılmıştır. Uyar ve ark. (Uyar, Şenli, \& Mutlu, 2012) beyin sinyallerini kullanarak protez kol kontrolü gerçekleştirmişlerdir. BCI (Brain Computer Interface) teknolojisi kullanarak beyin sinyallerini bilgisayar ortamına aktarmışlardır. Başın 16 farklı noktasına beyin dalga sensoru yerleştirerek EMG verileri elde etmişler ve dört hareket tanımlamışlardır. Bu hareketlerle protez kol kontrolünü gerçekleştirmişlerdir. Cognolato ve ark. (Cognolato, Atzori, Marchesini, et al., 2018) myo kol bandı kullanarak protez kolu gerçek zamanlı olarak kontrol etmişlerdir. Eli olmayan iki ampute birey kullanmışlardır. Myo kalibrasyonunda bulunan 4 el hareketini kullanarak 6 farklı nesneyi kavramaya çalışmışlardır. Protez el ile kavrama ve burakma hareketini gerçek zamanlı olarak oluşturmuş̧lardır. Abraham ve ark. (Abraham, Kwon, Solomon, Xie, \& Yeh, 2015) myo kol bandı kullanarak protez kolu arduino ile kontrol etmişlerdir. Myo bileklikte tanımlı el hareketlerini kullanarak protez kontrolünü gerçekleştirmişlerdir. Moneada ve ark. (Moneada, Satizabal, Hoyos, \& Padilla, 2017) myo kol bandında tanımlanan 5 el hareketini kullanarak arduino mega ile protez el kontrolü yapmışlardır.

Myo kol bandı kullanılarak yapılan protez ve robot kol çalışmalarında veriler kablosuz olarak aktarılmıştır. Robot kol, protez kol ve diğer çalışmalar myo bileklikte oluşturulan temel el hareketlerinden alınan veriler ile kontrol edilmiştir. Bu çalışmada ise tanımlanan el hareketleri ile Anahtarlama Mod Yöntemi (Heerschop, van der Sluis, Otten, \& Bongers, 2020) kullanılarak birçok farklı protez kol hareketi oluşturulmuş ve kontrol edilmiş̧ir. Böylece ampute bireylerde koldaki kısıtlı sayıdaki kas grupları kullanılarak farklı protez kol hareketleri gerçekleştirilmiştir. Bu da ampute bireylerin kısıtlı sayıdaki aktif olan kas grupları ile daha fazla sayıda protez el ve kol hareketlerinin kontrolüne imkan sağlamaktadır.

Makalenin giriş bölümünde myo kol bandı ile yapılan çalışmalar hakkında bilgi verilmiştir. Sistemin genel yapısı, materyallerin özellikleri ve kullanılan yöntem ikinci bölümde anlatılmaktadır. Makalenin üçüncü bölümünde bulgulara yer verilmiştir. Protez kolun kontrolü bu bölümde şekiller ve çizelgelerle birlikte detaylı olarak açıklanmıştır. Makalenin dördüncü kısmında tartışma bölümünden oluşmaktadır. Makalenin son bölümünde ise sonuçlara yer verilmiş yapılan çalışma hakkında kısa bir değerlendirme yapılmıştır. 


\section{Materyal ve Metot}

Bu çalışmada Anahtarlama Mod Yöntemi kullanılmıştır. Anahtarlama Mod yöntemi oluşturulan sistemlerde modlar arası geçiş için kullanılmaktadır. Bu yöntemin çalışma mantığı bir mod içerisinde iken o modu bitirme veya çıkış noktası belirlenir. Belirlenen çıkış noktasına gelindiğinde sistem o modun içerisinde çıkar ve sistem içerisindeki yeni moda girer. Böylece modlar arası geçiş sağlanır ve sonsuz döngü yapma imkanı oluşmaktadır. Bu yöntem bir döngü içerisindeki iş yükünü birkaç döngü oluşturarak diğerlerine paylaştırmamızı olanak sağlamaktadır. Ayrıca sınırlı sayıda giriş için farklı modlar oluşturarak sistemi genişletmemizi yardımcı olmaktadır.

Anahtarlama Mod Yöntemi kullanılarak modlar arası geçişler myo kol bandındaki 9 eksenli IMU içerisinde bulunan Jiroskop verileri ile gerçek zamanlı olarak gerçekleştirilmiştir. Sonrasında myo kol bandında tanımlanan dört el hareketi ve IMU verileri ile protez kol kontrol edilmiştir. Myo kol bandından alınan veriler HM 10 Bluetooth Modülü ile arduinoya, arduinodan protez kola aktarılmıştır. Protez kolun tasarımı el, bilek, dirsek ve omuz olmak üzere dört kısma ayrılır. El kontrolü için her parmak ayrı ayrı olmak üzere beş adet 0 ile 180 derece arasında değişen açılarla kontrol edilebilen servo motorlar kullanılarak kontrol edilmiştir. Bilek, dirsek ve omuz için birer adet servo motor kullanılmıştır. Protez kolda 8 adet servo motor kullanılmış ve protez kol 3 boyutlu yazıcıda oluşturulmuştur(Langevin). Oluşturulan sistemin genel yapısı ve kullanılan materyaller Hata! Başvuru kaynağı bulunamadı.'de gösterilmiştir.

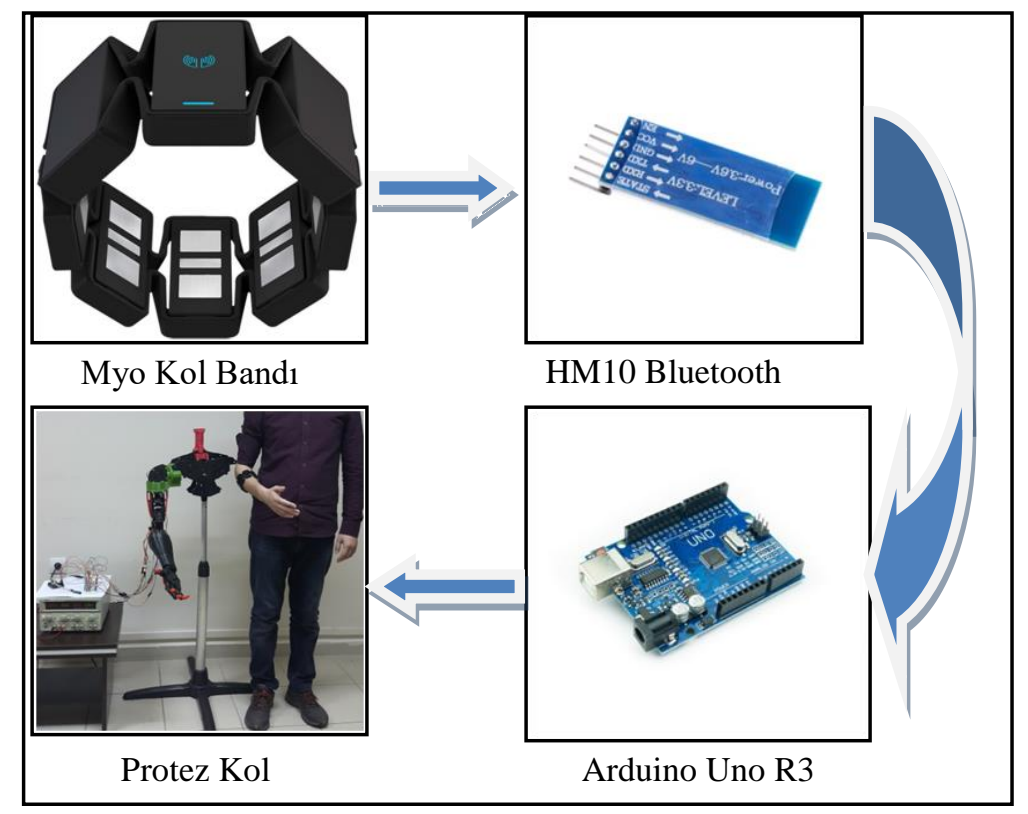

Şekil 1. Sistemin genel yapısl

\subsection{Myo Kol Bandı}

Myo bileklik kola takılarak kullanılan, kol üzerinde bulunan kasların hareketini algılayan ve Thalmics Labs. tarafından üretilen teknolojik bir cihazdır(North). Myo kol bandı Şekil 1'de gösterilmektedir.

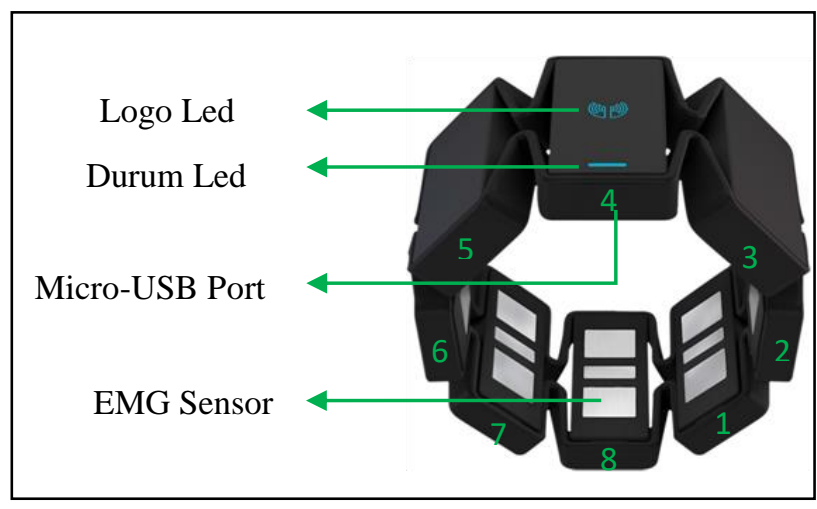

Şekil 1. Myo kol bandl

Myo içerisinde 8 adet EMG sensorları bulunmaktadır. Kasların kasılması sonucunda ortaya çıkan titreşimler EMG sensorları ile ölçülmektedir. Bu sensorları ile kasların kasılması sonucu veriler ölçülmektedir. Elin hangi hareketi yaptığı kasların kasılmasıyla yaptıkları titreşimler sonucunda EMG sensorları ile belirlenebilir. Ayrıca myo bileklik içerisinde son derece hassas dokuz eksenli IMU (Atalet Ölçüm Ünitesi) bulunmaktadır. IMU içerisinde 3 eksenli jiroskop, 3eksenli ivmeölçer ve 3 eksenli manyetometre 
bulunmaktadır. Myo içerisinde bulunan donanımlar ile el hareketleri ve elin konumu alınan verilerle hesaplanabilmektedir. Myo üzerinde ARM Cortex M4 işlemci bulunmaktadır. Myo bileklik bluetooth ile diğer cihazlara EMG ve IMU verilerini aktarmaktadır.

\subsection{HM10 Bluetooth Modülü}

$\mathrm{Bu}$ çalışmada myo bileklikten arduinoya veri aktarımı HM10 bluetooth modülü ile sağlanmıştır. Bu modül bluetooth 4.0 teknolojisine sahiptir. Düşük enerji harcayarak kablosuz iletişim sağlar. Çalışma frekansı $2.4 \mathrm{GHz}$ olup 30 metre haberleşme sağlamaktadır. $2.5 \mathrm{~V}$ ile $3.3 \mathrm{~V}$ arasında çalışma gerilimine sahiptir. $50 \mathrm{~mA}$ akım çekmektedir. $27 \mathrm{~mm} \times 13 \mathrm{~mm}$ x $2.2 \mathrm{~mm}$ boyutlarına sahiptir. HM10 Bluetooth Modülü Hata! Başvuru kaynağı bulunamadı.'te gösterilmiştir.

Bu çalışmada myo bilelik ile HM10 Bluetooth Modülünün bluetooth üzerinden haberleşebilmesi için sağlamak için HM10 Bluetooth Modülüne yazılım yüklenmiştir. Veri aktarımı bu şekilde gerçekleştirilmiştir. Kullanılan malzemelerim donanım bağlantıları Şekil 2'te gösterilmiştir. Arduino, HM 10 Bluetooth Modülü, sekiz adet servo motor ve $12 \mathrm{~V}$ ile $5 \mathrm{~A}$ güç verebilen güç kaynağının bağlantıları gösterilmiştir.

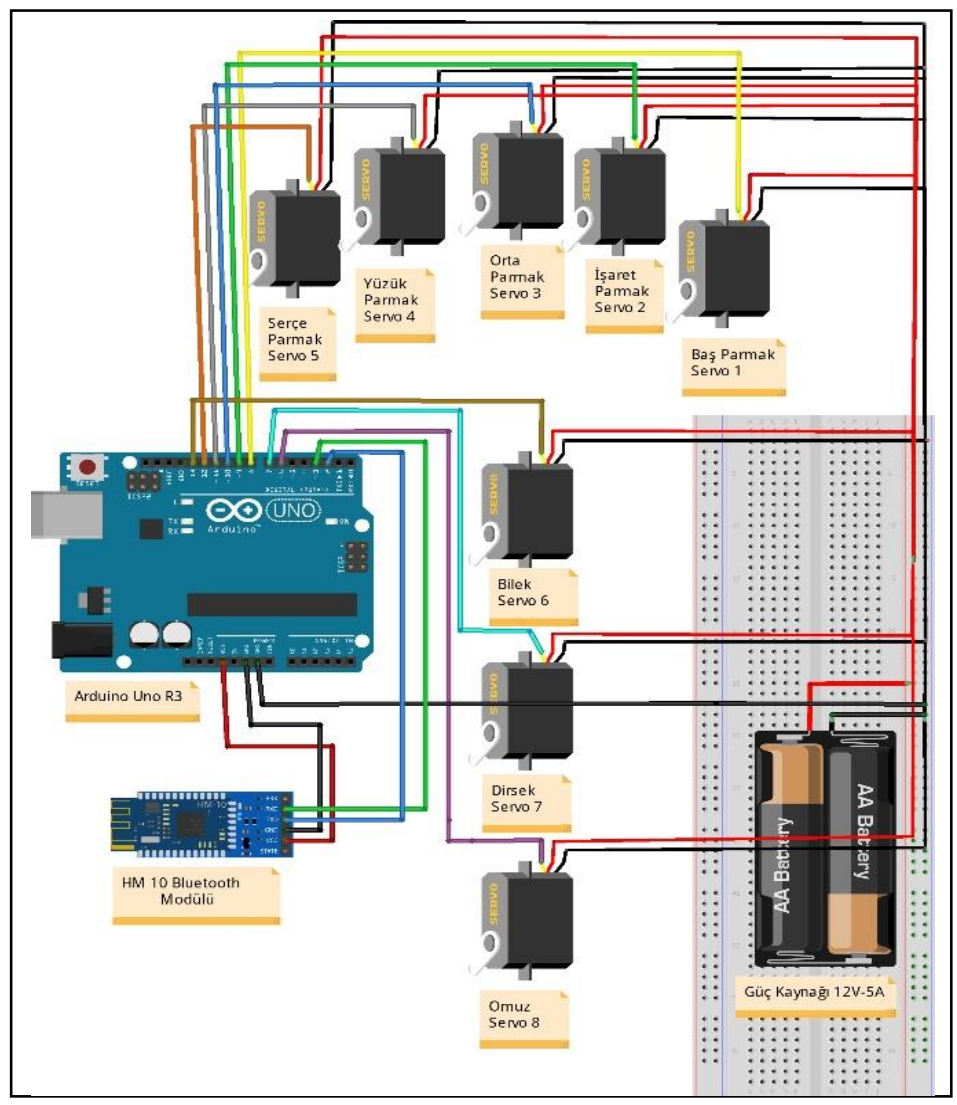

Şekil 2. Malzemelerin bağlantıları

$\mathrm{Bu}$ çalı̧̧ma için özel olarak Arduino uyumlu baskılı devre tasarlanmış ve gerçeklenmiştir. Bu devre üzerinde 8 adet servo motor bağlantısı, bluetooth modül bağlantısı, güç kaynağı bağlantısı ve arduino bağlantısı bulunmaktadır. Baskılı devre Şekil 3'te gösterilmektedir. Bu parça ile kablo karışıklığı önlenmiştir. Böylece malzemelerin bağlantıları daha düzgün ve kolay yapılmıştır.

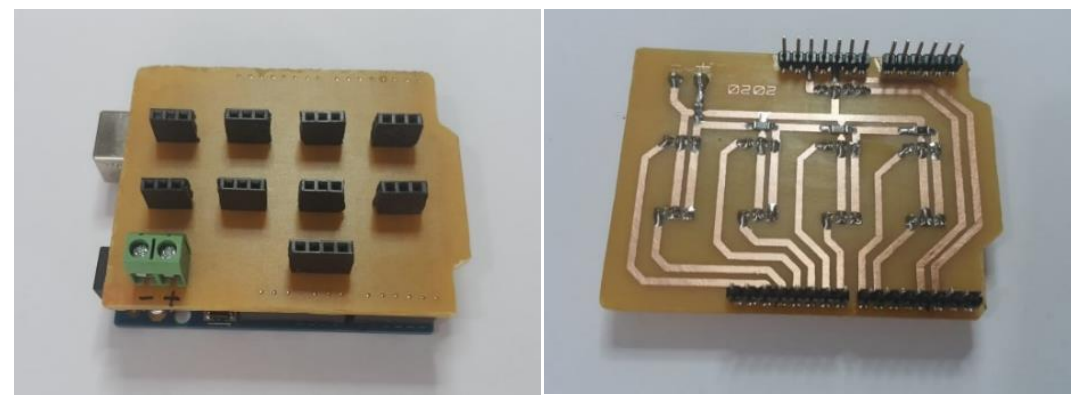

Şekil 3. Arduino uyumlu baskll devre

\subsection{Protez Kol Kontrolü}

$\mathrm{Bu}$ çalışmada el aç, el kapa, el içeri ve el dışarı hareketleri için myo bileklik kullanılarak 8 kanallı EMG verileri alınmıştır. Bu veriler Şekil 4'te gösterilmiştir. 


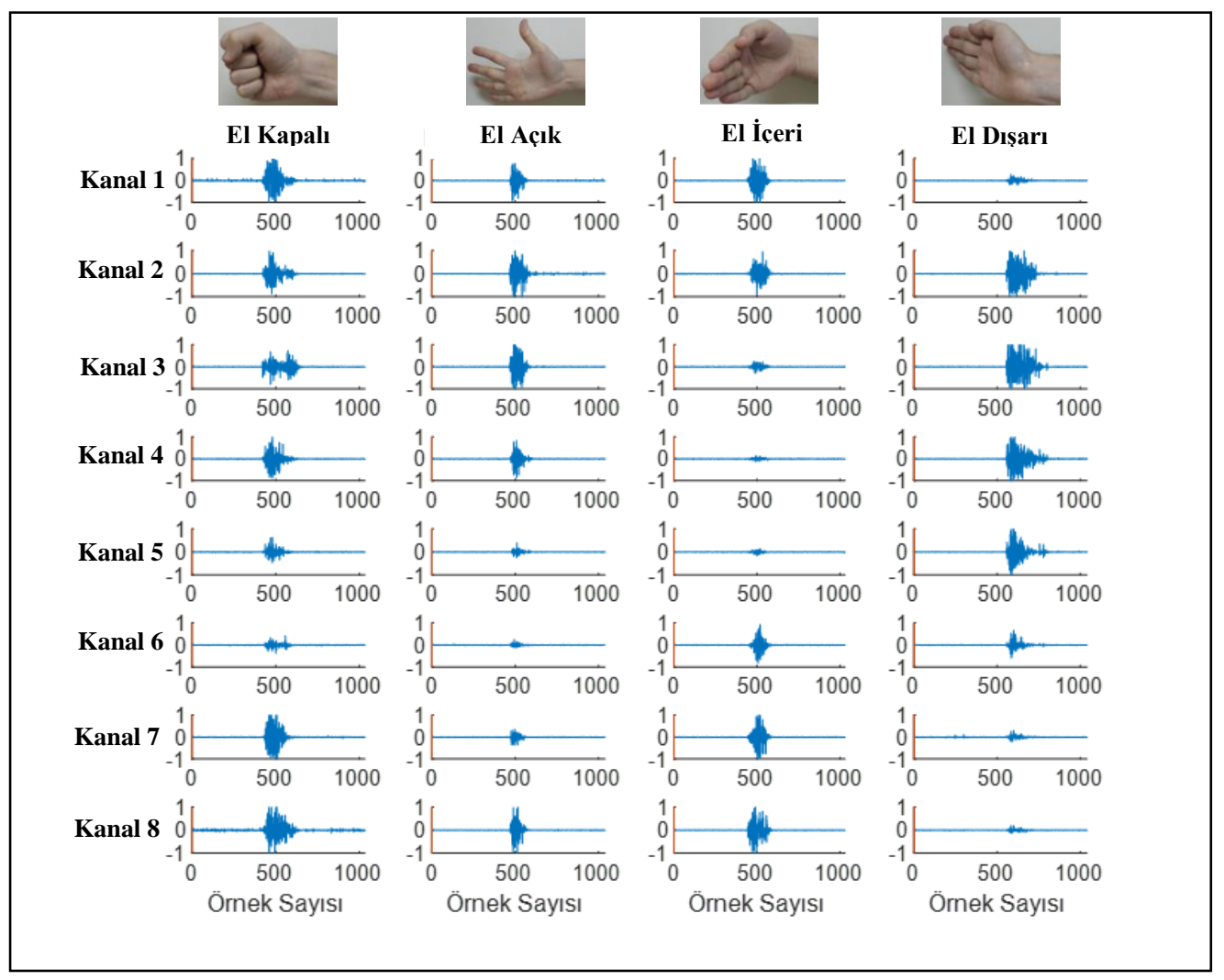

Şekil 4. Tanımlanan el hareketlerinin 8 kanallı emg verilerinin grafiği

Myo bileklikten verilerin hızlı ve doğru bir şekilde alınabilmesi amacıyla myo kol bandında bulunan kullanıcı ara yüzü kullanılmıştır. Bu ara yüz içerisinde kullanıcı kendine özel ara yüz oluşturabilmektedir. Ara yüz myo bileklik üreticisi Thalmics Labs. tarafından oluşturulmuş ve kullanıma sunulmuştur. Burada ara yüz oluşturularak myo kol bandı kalibrasyonu yapılır ve Şekil 5Hata! Başvuru kaynağı bulunamadı.'de gösterilmektedir. Myo bileklik böylece daha etkin ve doğru kullanılır.

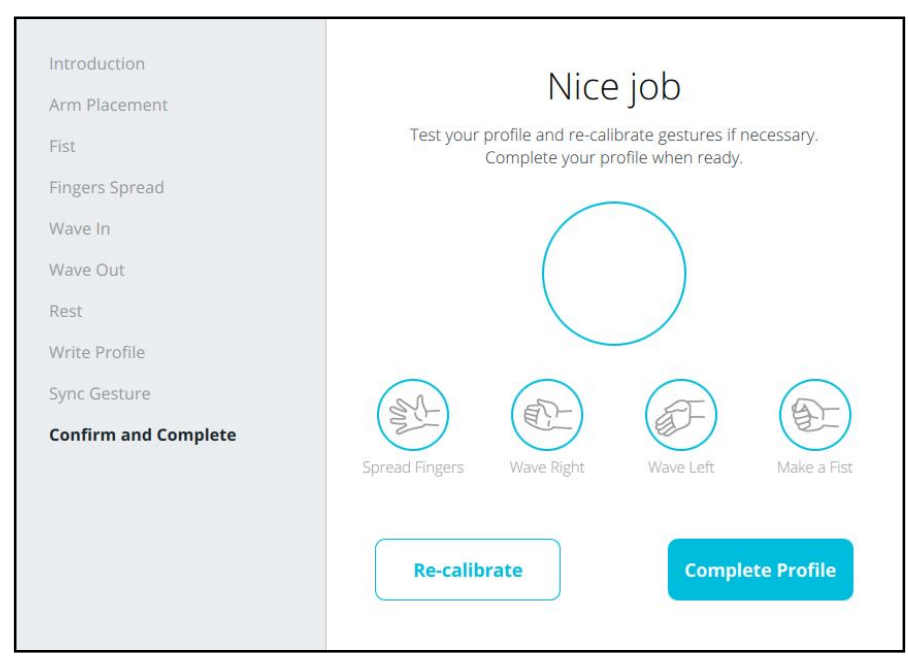

Şekil 5. Myo kol bandı kullanıcı kalibrasyonu

Tanımlanan bu ara yüz ile 4 el hareketin sınıflandırılması yapılmaktadır. Bu çalışmada dört adet döngü oluşturulmuştur. Döngüler el hareketleri, bilek, dirsek ve omuz hareketleri kontrol etmek için dört adet oluşturulmuştur. Döngü geçişleri myo bileklikten alınan jiroskop verileri ile gerçek zamanlı olarak sağlanmaktadır. Döngü geçişleri Anahtarlama Mod Yöntemi kullanılarak yapılmıştır. Bu yöntem sayesinde oluşturulan dört el hareketi ile protez kolun tamamı kontrol edilebilmiştir. Birçok hareketin gerçek zamanlı olarak yapılabilmesine olanak sağlamıştır. Kullanılan servo motorların protez kolda kullanılan tüm açı değerleri Tablo 1'de gösterilmiştir.

İlk 5 servo motor protez kolda bulunan parmakların kontrolü için kullanılmıştır. 6. servo motor bilek hareketleri için kullanılmıştır. 7. servo motor dirsek hareketleri için kullanılmıştır. Elden dirseğe kadar olan bölümde 7. servo motor ile yukarıya veya aşağıya doğru hareket ettirilmiştir. 8. Servo motor ile omuzdan hareket sağlanmıştır. Protez kol ortada, sağ tarafta veya sol tarafta olacak şekilde hareket ettirilmiştir. El kapalı hareketi için birinci, dördüncü ve beşinci servo motorlar 100 derece, ikinci ve üçüncü servo motor 120 derece olarak belirlenmiştir. El açık hareketi için birinci, ikinci, üçüncü, dördüncü ve beşinci servo motorlar 0 derece olarak belirlenmiştir. Başlangıç değeri sıfır olarak alınmış ve el açık pozisyonunda başlatılmıştır. Bilek içeri dön hareketi için altıncı servo motor 160 derece olarak belirlenmiştir. Bilek dışarı hareketi için altıncı servo motor 10 derece olarak belirlenmiştir. Barış 
hareketi için birinci, dördüncü ve beşinci servo motorlar 100 derece, ikinci ve üçüncü servo motorlar 0 derece olarak belirlenmiştir. Beni ara el hareketi için ikinci, üçüncü ve dördüncü servo motor 120 derece, birinci ve beşinci servo motor 0 derece olarak belirlenmiştir. Bu dereceler protez kolun konumuna göre belirlenmiştir.

Tablo 1. El hareketlerinde kullanılan servo motorların açı değgerleri

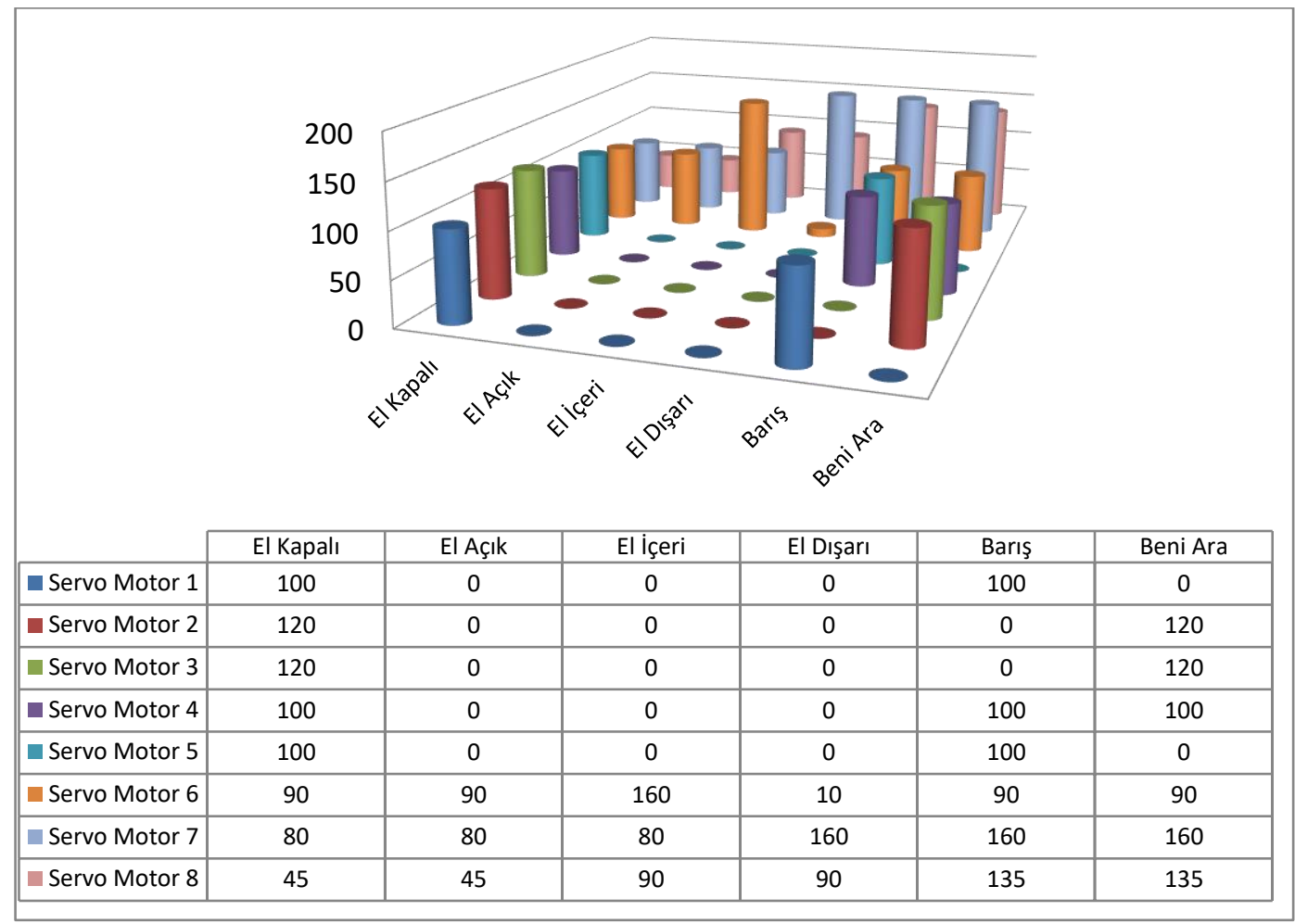

Tanımlanan el hareketleri ile yapılan protez kol kontrolleri Tablo 2‘de gösterilmektedir.

Tablo 2. Protez kol el hareketleri - işlev tablosu

\begin{tabular}{|c|c|c|c|c|}
\hline \multirow{3}{*}{ El Hareketleri } & \multicolumn{4}{|c|}{ Yapılan İşlemler } \\
\hline & Döngü 1 & Döngü 2 & Döngü 3 & Döngü 4 \\
\hline & $\begin{array}{c}\text { El } \\
\text { Hareketleri }\end{array}$ & $\begin{array}{c}\text { Bilek } \\
\text { Hareketleri }\end{array}$ & $\begin{array}{c}\text { Dirsek } \\
\text { Hareketleri } \\
\end{array}$ & $\begin{array}{c}\text { Omuz } \\
\text { Hareketleri }\end{array}$ \\
\hline & $\begin{array}{c}\text { El kapalı } \\
\text { hareketi } \\
\text { yapılır. }\end{array}$ & - & - & $\begin{array}{c}\text { Kol ortada } \\
\text { bulunur. }\end{array}$ \\
\hline & $\begin{array}{c}\text { Barış } \\
\text { hareketi } \\
\text { yapılır. }\end{array}$ & $\begin{array}{c}\text { Bilek sola } \\
\text { doğru } \\
\text { döner. }\end{array}$ & $\begin{array}{c}\text { Kol } \\
\text { aşağıya } \\
\text { hareket } \\
\text { eder. }\end{array}$ & $\begin{array}{c}\text { Kol sağ } \\
\text { tarafta } \\
\text { bulunur. }\end{array}$ \\
\hline & $\begin{array}{c}\text { Beni ara el } \\
\text { hareketi } \\
\text { yapilır. }\end{array}$ & $\begin{array}{c}\text { Bilek sağa } \\
\text { doğru } \\
\text { döner. }\end{array}$ & $\begin{array}{c}\text { Kol } \\
\text { yukarıya } \\
\text { hareket } \\
\text { eder. }\end{array}$ & $\begin{array}{c}\text { Kol sol } \\
\text { tarafta } \\
\text { bulunur. }\end{array}$ \\
\hline & $\begin{array}{c}\text { Parmaklar } \\
\text { ayrık el } \\
\text { hareketi } \\
\text { yapılır. }\end{array}$ & - & - & - \\
\hline
\end{tabular}

Protez kol denetimi akış şeması Şekil 6'de gösterilmiştir. Burada case0-3 Şekil 4'te gösterilen el hareketlerini temsil etmektedir(soldan sağa doğru). 


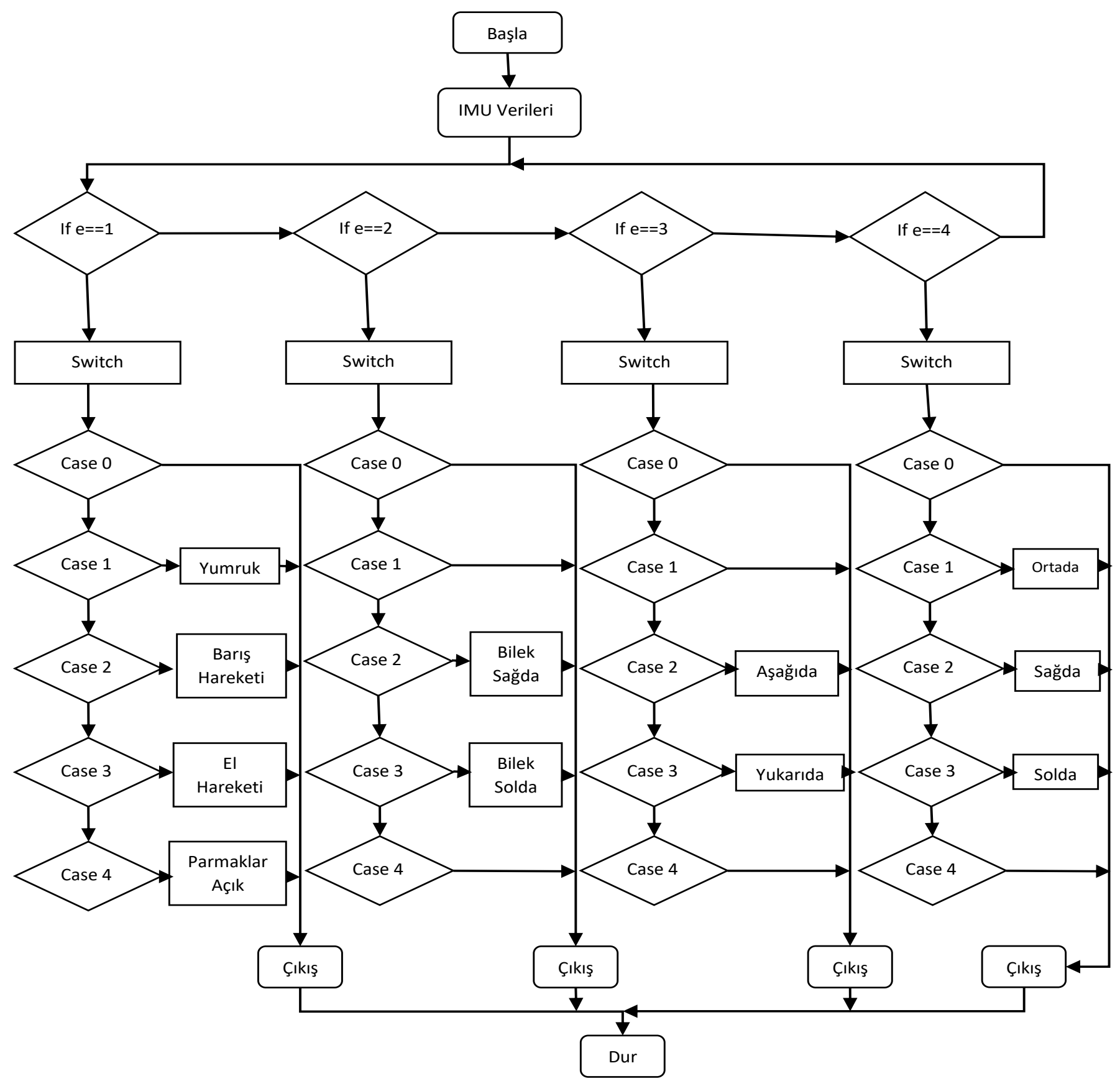

Şekil 6. Protez kol kontrolü akışşeması

\section{Araştırma Sonuçları}

Şekil 7'deki akış diyagramında gösterildiği gibi myo bileklikten verilerini okur ve Şekil 7 deki akış diyagramına göre ilk döngüye girer. Birinci döngüde myo kol bandında tanımlanan el hareketlerini kullanır. Birinci döngüde el aç, el kapa, barış ve beni ara el hareketi olmak üzere 4 el hareketi tanımlanmıştır. Bu el hareketleri kümesi ile gerçek zamanlı olarak başarılı bir şekilde protez el denetimi (el aç, el kapa, barış ve telefon hareketleri ) yapılmıştır. Yapılan hareketler Şekil 7'de gösterilmiştir. 

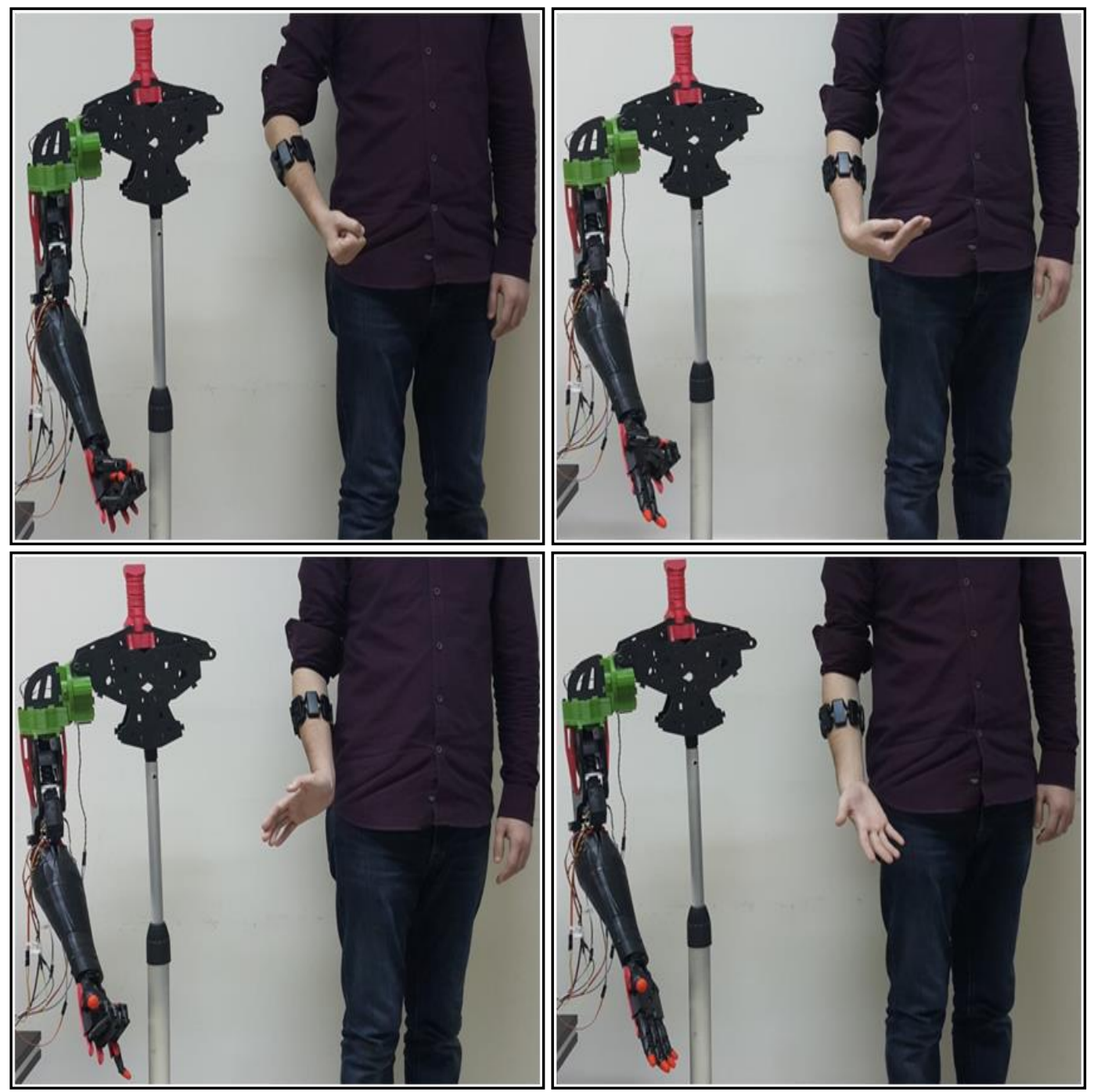

Şekil 7. Birinci döngüdeki kol ile protez kol hareketleri

Döngü geçişleri omuzdan yukarıya ve aşağıya, hızlı ve tek bir hareket sonucunda gerçekleşmektedir. Bu hareket sonucunda jireskop verilerinde ani bir değişiklik oluşmaktadır. Arduino ile myo bileklik içerisinde bulunan üç eksenli jiroskop verileri okunmaktadır. Bu veriler üzerinde eşikleme yapılarak döngü geçişleri yapılmaktadır. İkinci döngüde bilek içeri ve bilek dışarı olmak üzere iki bilek hareketi tanımlanmıştır. Bu bilek hareketleri kümesi ile gerçek zamanlı olarak başarılı bir şekilde protez bilek hareketleri (bilek içeri, bilek dışarı) denetimi yapılmıştır Bilek hareketleri Şekil 8'da gösterilmiştir.
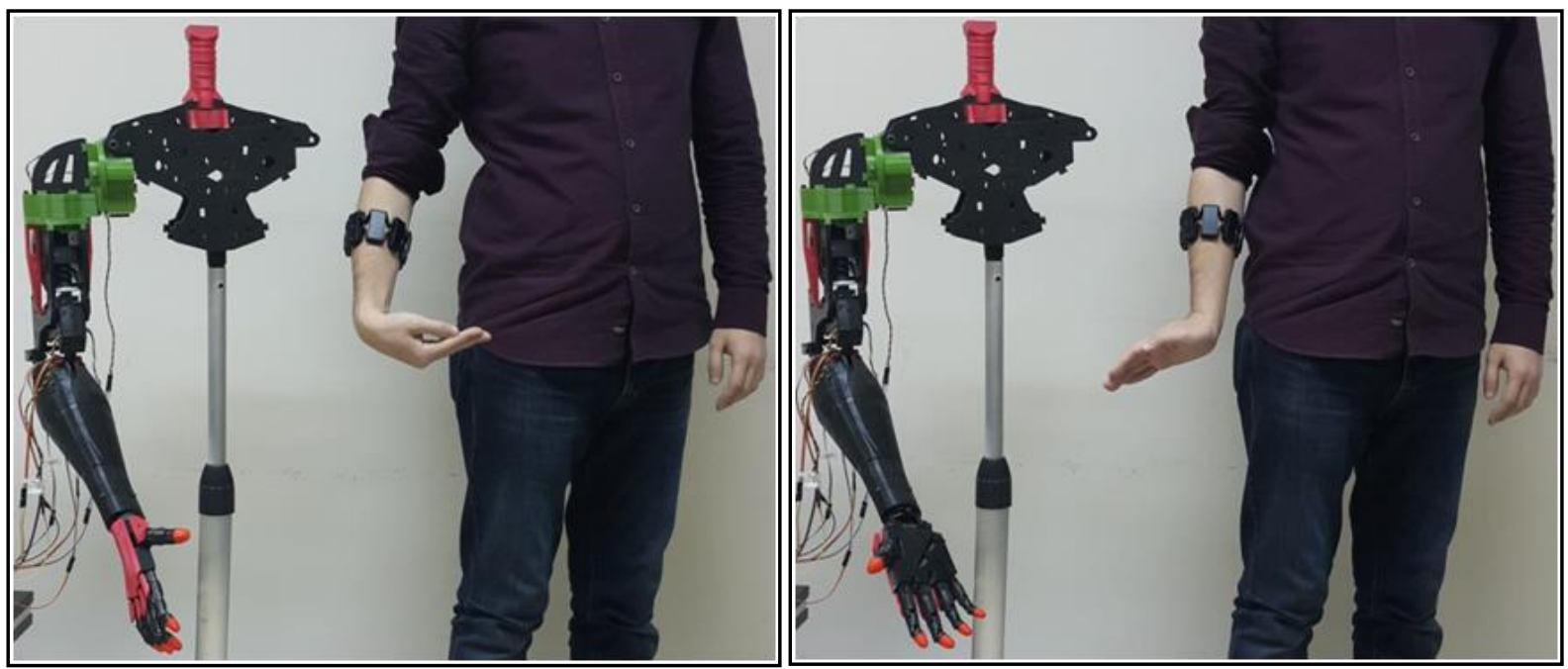

Şekil 8. Íkinci döngüdeki bilek hareketleri 
Üçüncü döngüde protez kolun dirsek aşağı ve yukarı yönlü hareket kontrolü yapılmıştır. Bu kontrol için el içeri ve el dışarı hareket kümesi kullanılmıştır. El içeri hareketi yapıldığında protez kol dirsekten aşağı yönlü, el dışarı hareketi yapıldığında ise protez kol dirsekten yukarı yönlü hareket etmektedir. Protez kol dirsek hareketleri Şekil 9'de gösterilmiştir.
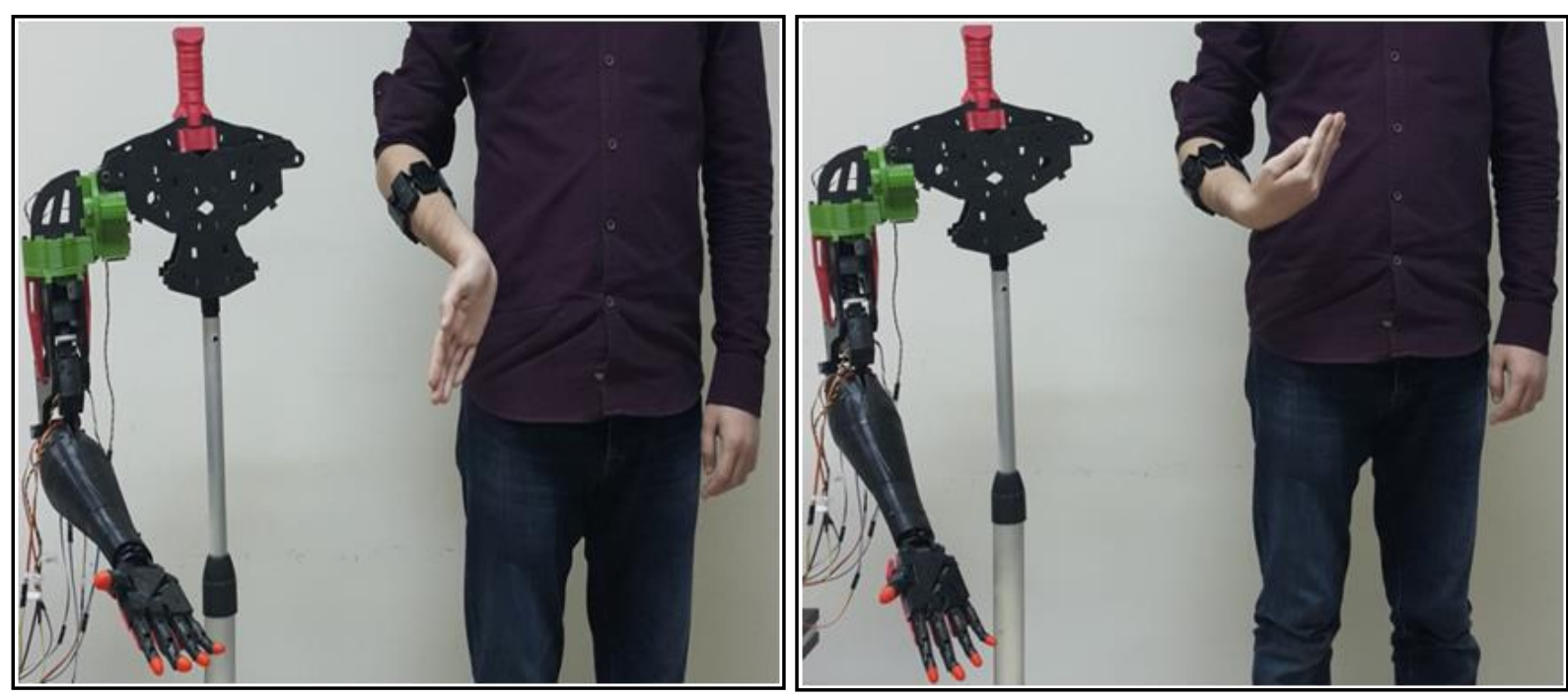

Şekil 9. Üçüncü döngüdeki dirsek hareketleri

Dördüncü döngüde ise protez kolda omuz hareketleri kontrol edilmektedir. Protez kol omuz hareketleri el kapalı, el içeri ve el dışarı hareket kümesi ile kontrol edilmiştir. El kapalı hareketi yapıldığında protez kol pozisyonu omuzdan itibaren orta kısımdadır. El içeri hareketi yapıldığında protez kol omuzdan dışarıya doğru açılmaktadır. El içeri hareketi yapıldığında protez kol omuzdan içeriye doğru hareket etmektedir. Omuz hareketleri Şekil 10'de gösterilmektedir.
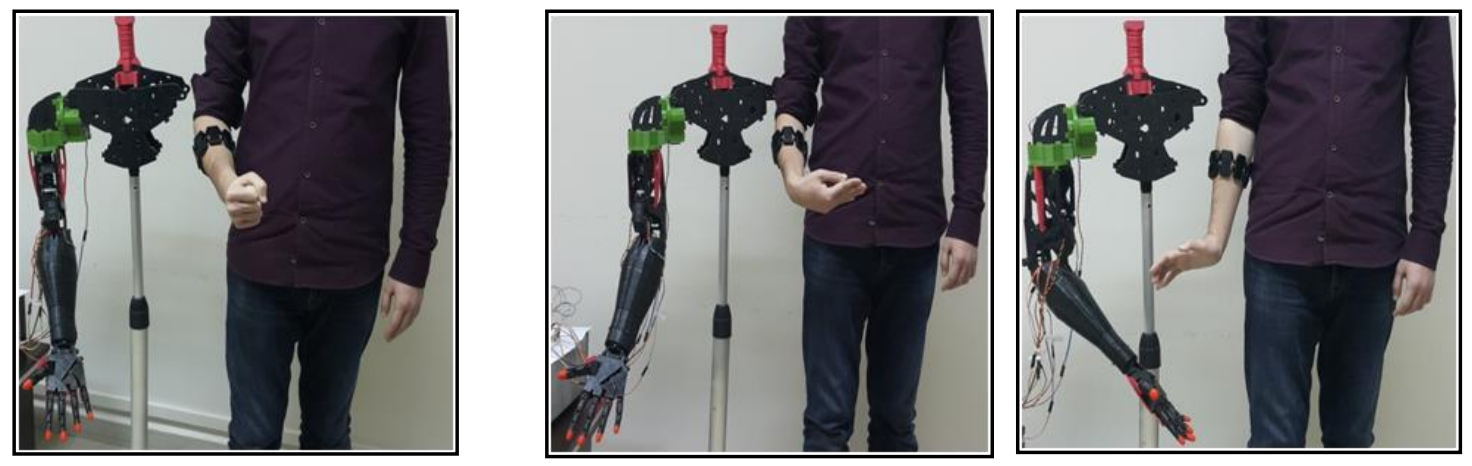

Şekil 10. Dördüncü döngüdeki omuz hareketleri

\section{SONUÇ VE TARTIŞMA}

$\mathrm{Bu}$ araştırmada insan kolu üzerine yerleştirilen myo kol bandı ile alınan 8 kanallı EMG işaretleri ve jiroskop verilerini kullanarak gerçek zamanlı protez kol kontrolü gerçekleştirilmiştir. Robot kol (Murillo \& Moreno, 2016; Ülkir et al., 2017) ve protez kol (Abraham et al., 2015; Cognolato, Atzori, Marchesini, et al., 2018; Moneada et al., 2017; Said et al., 2019) üzerine myo bileklik kullanarak benzer çalışmalar yapılmıştır. Bu çalışmada ise literatürde bulunan benzer çalışmadan farklı olarak myo kol bandından alınan veriler Anahtarlama Mod Yöntemi ile birçok döngü oluşturulmuştur. Döngü geçişleri için herhangi bir el hareketine gerek duyulmadan jiroskop verileri ile gerçekleştirilmiştir. Kullanılan bu yöntem ile ampüte bireylerdeki kısıtlı sayıdaki kas gruplarıyla çok fazla sayıda protez kol hareketlerinin (el, bilek, dirsek ve omuz) gerçek zamanlı olarak kontrolü gerçekleştirilmiştir. Literatür incelendiğinde herhangi bir çalışmada böyle bir sonuca rastlanmamıştır, bu durumun çalışma sonucunun literatürdeki boşluğun doldurulmasına katkı sağladığı söylenebilir.

Literatürdeki çalışmalarda sadece protez el ve bilek kontrolü yapılmıştır (Abraham et al., 2015; Cognolato, Atzori, Marchesini, et al., 2018; Moneada et al., 2017; Said et al., 2019). Bu çalışmada ise protez el, bilek, dirsek ve omuz kontrolü yapılmıştır.

Ayrıca bluetooth modülü ile doğrudan mikro denetleyiciye aktarılarak taşınabilir bir sistem geliştirilmiştir. Literatürdeki bazı çalışmalarda bilgisayarda veriler işlenerek kol kontrol edilmiş̧ir(Abraham et al., 2015; Cognolato, Atzori, Marchesini, et al., 2018; Murillo \& Moreno, 2016; Said et al., 2019). Bu çalışmada bilgisayara herhangi bir veri aktarımı olmamıştır. Bu da bilgisayara ihtiyaç duyulmadan kontrol yapabilme avantajı sağlamıştır.

Literatürdeki çalışmalarda kablolama dağınık bir şekilde yapılmıştır(Abraham et al., 2015; Moneada et al., 2017; Murillo \& Moreno, 2016; Ülkir et al., 2017). Bu çalışmada ise arduino üzerine eklenebilen baskı devre kartı oluşturulmuş kullanılan malzemelerin bağlantıları bu devre üzerinden gerçekleştirilmiş̧ir. Bu devre ile kablo karmaşasına son verilmiş düzgün bir görünüm 
sağlanmıştır. Bağlantılar daha kolay yapılabilmiştir. Oluşturulan sistem gerçek zamanlı olarak protez kol kontrollerinde kullanılabilir yapıdadır. Bu çalışma gerçek protez kol sistemlerine uygulanarak başarımı ampute bireyler üzerinde test edilebilir.

\section{Kaynakça}

Abraham, Z., Kwon, D. B., Solomon, T., Xie, M., \& Yeh, K. (2015). Control of an affordable hand and wrist prosthesis. Paper presented at the 15th Research Symposium, Rutgers School of Engineering.

Boyali, A., \& Hashimoto, N. (2016). Spectral Collaborative Representation based Classification for hand gestures recognition on electromyography signals. Biomedical Signal Processing Control, 24, 11-18.

Cacace, J., Finzi, A., \& Lippiello, V. (2016). Multimodal interaction with multiple co-located drones in search and rescue missions. arXiv preprint arXiv:1605.07316.

Cognolato, M., Atzori, M., Faccio, D., Tiengo, C., Bassette, F., Gassert, R., \& Muller, H. (2018). Hand Gesture Classification in Transradial Amputees Using the Myo Armband Classifier. Paper presented at the 2018 7th IEEE International Conference on Biomedical Robotics and Biomechatronics (Biorob).

Cognolato, M., Atzori, M., Marchesini, C., Marangon, S., Faccio, D., Tiengo, C., . . Müller, H. (2018). Multifunction control and evaluation of a 3D printed hand prosthesis with the Myo armband by hand amputees. BioRxiv, 445460.

Çoban, M., \& Gelen, G. (2018). Wireless teleoperation of an industrial robot by using myo arm band. Paper presented at the 2018 International Conference on Artificial Intelligence and Data Processing (IDAP).

Erin, K., \& Boru, B. (2018). EMG ve jiroskop verileri ile endüstriyel robot kolunun gerçek zamanlı kontrolü. Sakarya University Journal of Science, 22(2), 509-515.

Ganiev, A., Shin, H.-S., \& Lee, K.-H. (2016). Study on virtual control of a robotic arm via a myo armband for the selfmanipulation of a hand amputee. Int. J. Appl. Eng. Res, 11(2), 775-782.

Gelen, G., \& Özcan, S. (2019). İnsan-robot etkileşiminin biyomimetik yaklaşımla sağlanması. Pamukkale Üniversitesi Mühendislik Bilimleri Dergisi, 25(2), 188-198.

Hassan, H. F., Abou-Loukh, S. J., \& Ibraheem, I. K. (2019). Teleoperated robotic arm movement using electromyography signal with wearable Myo armband. Journal of King Saud University-Engineering Sciences.

Heerschop, A., van der Sluis, C. K., Otten, E., \& Bongers, R. M. (2020). Looking beyond proportional control: The relevance of mode switching in learning to operate multi-articulating myoelectric upper-limb prostheses. Biomedical Signal Processing Control, 55, 101647.

Klein, B. (2015). A Gesture Control Framework Targeting High-Resolution Video Wall Displays. Paper presented at the 2015 19th International Conference on Information Visualisation.

Kristof, R., Moldovan, C., Ciupe, V., Maniu, I., \& Banda, M. (2019). Applications based on electromyography sensors. Paper presented at the ITM Web of Conferences.

Langevin, G. (20.02.2020). Prosthetic Hand. from http://inmoov.fr/

Lopes, J., Simão, M., Mendes, N., Safeea, M., Afonso, J., \& Neto, P. (2017). Hand/arm gesture segmentation by motion using IMU and EMG sensing. Procedia Manufacturing, 11, 107-113.

Moneada, A., Satizabal, D., Hoyos, G., \& Padilla, B. (2017). Integration of a prototype of bionic prosthesis hand. Paper presented at the 2017 IEEE 3rd Colombian Conference on Automatic Control (CCAC).

Morais, G. D., Neves, L. C., Masiero, A. A., \& de Castro, M. C. F. (2016). Application of Myo Armband System to Control a Robot Interface. Paper presented at the BIOSIGNALS.

Murillo, P. U., \& Moreno, R. J. (2016). Individual robotic arms manipulator control employing electromyographic signals acquired by myo armbands. Int. J. Appl. Eng. Res, 11(23), 11241-11249.

North. (20.12.2019). from https://support.getmyo.com/hc/en-us

Said, S., Sheikh, M., Al-Rashidi, F., Lakys, Y., Beyrouthy, T., \& Nait-ali, A. (2019). A Customizable Wearable Robust 3D Printed Bionic Arm: Muscle Controlled. Paper presented at the 2019 3rd International Conference on Bio-engineering for Smart Technologies (BioSMART).

Sathiyanarayanan, M., \& Rajan, S. (2016). MYO Armband for physiotherapy healthcare: A case study using gesture recognition application. Paper presented at the 2016 8th International Conference on Communication Systems and Networks (COMSNETS).

Tabor, A., Kienzle, A., Smith, C., Watson, A., Wuertz, J., \& Hanna, D. (2016). The Falling of Momo: A Myo-Electric Controlled Game to Support Research in Prosthesis Training. Paper presented at the Proceedings of the 2016 Annual Symposium on Computer-Human Interaction in Play Companion Extended Abstracts.

Uyar, E., Şenli, K., \& Mutlu, L. (2012). Beyin Dalgası Kontrollü Protez Kol Tasarımı. Sakarya University Journal of Science, 16(3), 164-169.

Uzunhisarcıklı, E., Çetinkaya, M. B., Fidan, U., \& Çalıkuşu, İ. (2019). Investigation of EMG Signals in Lower Extremity Muscle Groups During Robotic Gait Exercises. Avrupa Bilim ve Teknoloji Dergisi, 109-118.

Ülkir, O., Akgün, G., \& Kaplanoğlu, E. (2017). Real time robotic arm control using wearable gesture armband. Paper presented at the Proc. 2nd International Mediterranean Science and Engineering Congress (IMSEC 2017).

Wopereis, H. W., Fumagalli, M., Stramigioli, S., \& Carloni, R. (2015). Bilateral human-robot control for semi-autonomous UAV navigation. Paper presented at the 2015 IEEE/RSJ International Conference on Intelligent Robots and Systems (IROS).

Xu, Y., Yang, C., Liu, X., \& Li, Z. (2018). A Teleoperated Shared Control Scheme for Mobile Robot Based sEMG. Paper presented at the 2018 3rd International Conference on Advanced Robotics and Mechatronics (ICARM).

Yüzgeç, U., Büyüktepe, H. E., \& Karakuzu, C. (2016). Kablosuz eldiven sistemi ile kontrol edilen robot kol tasarımı. Türkiye Bilişim Vakfi Bilgisayar Bilimleri ve Mühendisliği Dergisi, 9(2), 35-42. 